PREPARED FOR THE U.S. DEPARTMENT OF ENERGY, UNDER CONTRACT DE-AC02-76CH03073

PPPL-3857

PPPL-3857

UC-70

Subcyclotron Instability of Alfvén Eigenmodes due to Energetic lons in Low Aspect Ratio Plasmas

by

N.N. Gorelenkov, E. Fredrickson, E. Belova, C.Z. Cheng, D. Gates, and R. White

August 2003

$N_{\substack{\text { PRInCETOn PLASMA } \\ \text { PHYSIES LABORATORY }}}^{D}$

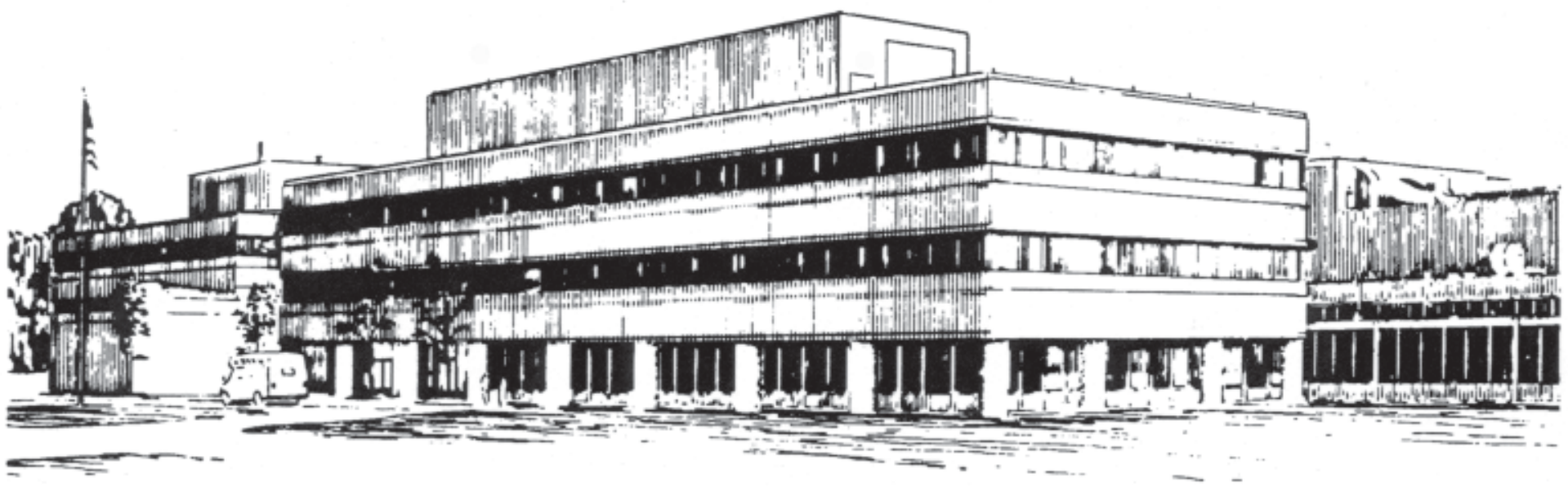

PRINCETON PLASMA PHYSICS LABORATORY PRINCETON UNIVERSITY, PRINCETON, NEW JERSEY 


\section{PPPL Reports Disclaimer}

This report was prepared as an account of work sponsored by an agency of the United States Government. Neither the United States Government nor any agency thereof, nor any of their employees, makes any warranty, express or implied, or assumes any legal liability or responsibility for the accuracy, completeness, or usefulness of any information, apparatus, product, or process disclosed, or represents that its use would not infringe privately owned rights. Reference herein to any specific commercial product, process, or service by trade name, trademark, manufacturer, or otherwise, does not necessarily constitute or imply its endorsement, recommendation, or favoring by the United States Government or any agency thereof. The views and opinions of authors expressed herein do not necessarily state or reflect those of the United States Government or any agency thereof.

\section{Availability}

This report is posted on the U.S. Department of Energy's Princeton Plasma Physics Laboratory Publications and Reports web site in Fiscal Year 2003. The home page for PPPL Reports and Publications is: http://www.pppl.gov/pub_report/

DOE and DOE Contractors can obtain copies of this report from:

U.S. Department of Energy

Office of Scientific and Technical Information

DOE Technical Information Services (DTIS)

P.O. Box 62

Oak Ridge, TN 37831

Telephone: (865) 576-8401

Fax: (865) 576-5728

Email: reports@adonis.osti.gov

This report is available to the general public from:

National Technical Information Service

U.S. Department of Commerce

5285 Port Royal Road

Springfield, VA 22161

Telephone: $1-800-553-6847$ or

(703) $605-6000$

Fax: (703) 321-8547

Internet: http://www.ntis.gov/ordering.htm 


\title{
Sub-cyclotron Instability of Alfvén Eigenmodes due to Energetic Ions in Low Aspect Ratio Plasmas ${ }^{1}$
}

\author{
N. N. Gorelenkov 1), E. Fredrickson 1), E. Belova 1), C. Z. Cheng 1), \\ D. Gates 1), R. White 1) \\ 1) Princeton Plasma Physics Laboratory, P.O. Box 451, Princeton, NJ, USA 08543-0451
}

\section{Observations}

Magnetic field activity measured by edge Mirnov coils during NBI injection in NSTX shows a broad and complicated frequency spectrum of coherent modes between $400 \mathrm{kHz}$ and up to $2.5 \mathrm{MHz}$, with the fundamental cyclotron frequency of background deuterium ions $f_{c D}=$ $\omega_{c D} / 2 \pi=2.3 \mathrm{MHz}$, calculated at the vacuum magnetic field at the geometrical axis of the plasma $B_{g 0}=0.3 T[1,2,3,4]$. The instability frequency spectrum has discrete peaks as shown in FIG. 1. Frequency spectrum peaks scale with the characteristic Alfvén velocity as magnetic field and plasma density are varied. NSTX is a low aspect ratio toroidal device with major and minor radii $R_{g 0}=0.85 m$ and $a=0.65 m$ respectively. In NSTX the Alfvén speed is low compared to the injection velocity of $E_{b 0} \simeq 80 \mathrm{keV}$

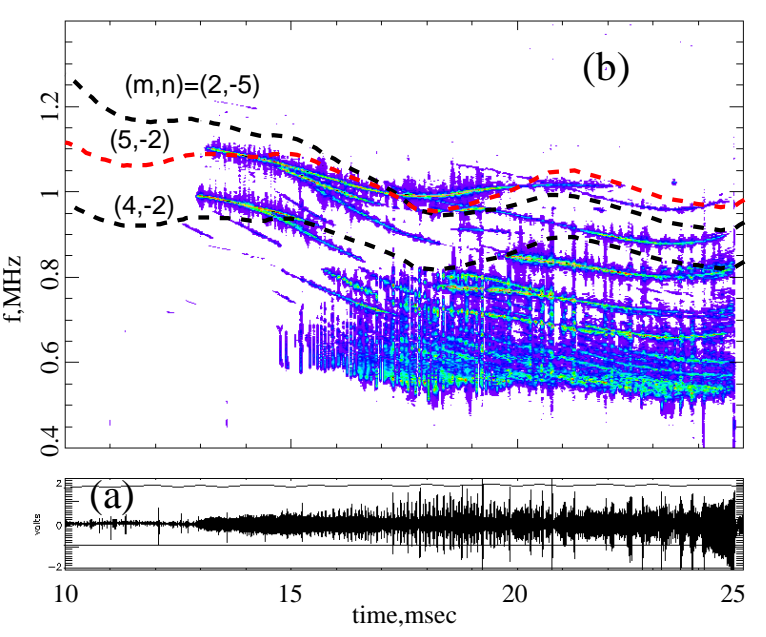

FIG. 1: Time evolution of the Mirnov signal (a), and its frequency spectrum (b) in NSTX shot \#108236.

NBI deuterium ions, with $v_{A} / v_{b 0} \simeq 1 / 4$. The power available to sustain these modes may be a fraction of the total auxiliary heating power larger than in conventional tokamaks and thus stronger Alfvén type instabilities are expected in NSTX.

For the sub-cyclotron frequency instabilities under consideration, the plasma allows for the shear Alfvén and compressional Alfvén (or magnetosonic) branches of oscillations. For the purpose of application to the low aspect ratio plasma of Spherical Tokamaks (ST) we developed a theory of radially and poloidaly localized Compressional Alfvén Eigenmodes (CAE) $[3,5]$. CAEs were studied earlier in connection with the problem of ion cyclotron emission in tokamaks $[6,7,8,9,10,11,12]$. Initially observed instabilities were identified as CAEs driven by energetic beam ions, since the predicted CAE frequency spectra were in agreement with the experiments, in which the magnetic signal peaks evolve parallel to each other. CAE frequencies are determined primarily by the Alfvén frequency at the mode location and the poloidal wave vector: $\omega_{C A E}=v_{A} m / r$, where $m$ is the poloidal mode number, and $r$ is the minor radius. In this paper we show additional evidence which helps to identify the observed instabilities as compressional Alfvén.

In new observations such as shown in FIG. 1 some spectrum peak lines intersect, indicating a more complicated dispersion than reported earlier for CAEs. We suggest that such modes are GAE's [13]. GAE's were found to be unstable in the nonlinear Hybrid MHD (HYM) code [14] modified for the ST geometry (see E. Belova paper this meeting). In conventional tokamaks GAE's were considered stable against the pressure driven instability given by fast particles as a result of strong continuum damping. This is due to the toroidal coupling to the kinetic mode at the edge [16]. In this paper we show that the continuum damping of GAEs is small for moderate to high mode numbers.

\footnotetext{
${ }^{1}$ This work supported by DoE contract No. DE-AC02-76CH03073
} 


\section{Compressional Alfvén Eigenmodes}

For a typical low aspect ratio plasma of NSTX the dispersion of CAEs can be presented in the form [5]

$$
\begin{aligned}
\omega_{m s n}^{2} & \simeq \frac{v_{A 00}^{2}}{r_{0}^{2}}\left\{\frac{4(m+1 / 2)^{2}}{\kappa^{2}}\left(\epsilon_{0}-\alpha_{0}\right)+\frac{\kappa^{4}-1}{\kappa^{4}} \frac{m^{2}+m+3 / 2}{2}\right. \\
& \left.+\frac{2(2 s+1)(2 m+1)}{\kappa} \sqrt{\frac{\left(\epsilon_{0}-\alpha_{0}\right)(1+\sigma)}{2 \sigma}}+n^{2}\left[\frac{q^{2}\left(r_{0}\right)}{\kappa^{2}}+\frac{R_{0}^{2}+4 r_{0}^{2}}{4 R_{00}^{2}}\right]\right\} .
\end{aligned}
$$

where $m, n$, and $s$ are the poloidal, toroidal and radial wave numbers, $\alpha_{0} \simeq B_{\theta}^{2} / 2 B_{\varphi}^{2}$ is the parameter which changes the depth of the potential well for the eigenmode due to the poloidal field, $r_{0}=1 / \sqrt{1+\sigma}, \kappa$ is the ellipticity, and the plasma density profile was chosen as $n_{e}=n_{e 0}\left(1-r^{2} / a^{2}\right)^{\sigma}$. The double subscript refers to the low field side point at $r=r_{0}$. To the lowest order in $m \gg 1$ the mode is localized at the low field side with a poloidal width of $\Theta=1 / \sqrt{\epsilon_{0}-\alpha_{0}}$. Radially the CAE is localized within the domain $\Delta^{2} / r_{0}^{2}=\kappa \sqrt{2 \sigma /(1+\sigma)\left(\epsilon_{0}-\alpha_{0}\right)} /(2 m+1)$. Note that $m$ is a quantum number and becomes the cylindrical poloidal mode number in the limit of high $m$ 's and high aspect ratio $[5,3]$. Spacing between the discrete peaks of the observed CAE frequency spectra is in agreement with theory. In discharges with pure CAEs the radial mode number corresponds to a frequency spacing on the order of $\Delta f_{s} \simeq 1 M H z$ and is responsible for two bands of CAE peaks at around $f \simeq 0.9 \mathrm{MHz}$ and $f \simeq 1.8 \mathrm{MHz}$. Within each band peaks are typically separated by $\Delta f_{m} \simeq 100-150 \mathrm{kHz}$ corresponding to neighboring poloidal mode numbers $m$ and $m+1$. Toroidal mode numbers produce the finest splitting of each frequency peak by $\Delta f_{n} \simeq 10-20 k H z$. These features were observed in experiments [1]. In addition a new analysis with the array of toroidally displaced coils shows that CAEs are compressional oscillations. As an example we consider one of the peaks on the Mirnov coil spectrum in NSTX discharge $\# 108236$ at $t=0.35 \mathrm{sec}$ which has frequency $f=1079 \mathrm{kHz} \simeq\left(\omega_{c} / 2 \pi\right) / 2$. The toroidal and poloidal components of the equilibrium magnetic field inside the last closed magnetic surface are $B_{\theta} / B_{\varphi}=0.67$, whereas the perturbed components were measured $\delta B_{\|}=0.66 \mathrm{mGauss}$, $\delta B_{\perp}=0.15 m$ Gauss. Note that in finite beta plasma compressional and shear branches are always coupled.

\section{Global shear Alfvén Eigenmodes}

GAE's are formed just below the minimum of the Alfvén continuum $\omega \simeq \pm \omega_{\text {Amin }}, \omega_{\text {Amin }}=$ $\left(k_{\|}(r) v_{A}(r)\right)_{\min }$ [13]. The GAE eigenfrequency is slightly shifted downward from $\omega_{A m i n}$, and the shift depends on the $q$ and density profiles. GAE is localized radially at the minimum of $\omega_{\text {Amin }}\left(r_{0}\right)$ and is dominated by one poloidal harmonic $m$. With the typically flat $q$ profile the Alfvén continuum has a minimum at the plasma center, so that $\omega \simeq \pm v_{A 0}\left(m / q_{0}-n\right) / R_{a x}$, where $R_{a x}$ is the major radius of the magnetic axes. One can see that if $q$ is evolving in time, the eigenfrequencies of GAE's with different combinations of $(m, n)$ will result in different evolutions. This can be seen from FIG. 1, where the instability peaks intersect. In FIG. 1 we plotted several simplified GAE eigenfrequencies $\omega \simeq-\omega_{\text {Amin }}$. The negative sign of the mode frequency is chosen to match the calculated frequency spectrum with the observed with toroidal mode numbers $|n|=4-5$ and to satisfy the resonance condition of co-injected beam ions with GAE's (see next section). Some discrepancy with the measured frequencies are due to uncertainties in the measurements of the plasma parameters and in the reconstruction of the equilibrium with the EFIT code. We have calculated the AE continuum and one of the GAE's (with $(m, n)=(-5,2))$ using the ideal MHD code NOVA [15] (see details in [4]). The frequency of this mode in the laboratory frame is $f=\left(1.18+n f_{\text {rot }}\right) M H z$, where $f_{\text {rot }} \simeq 11 \mathrm{kHz}$ accounts for the plasma rotation. This frequency closely (within $5 \%$ ) matches the observed frequency. The frequency separation between the different modes is primarily due to changes in $n$ or $m$, so that as NOVA predicts $\Delta f=f_{m, n-1}-f_{m, n}=v_{A 0} / R-f_{r o t}=175 \mathrm{kHz}$ 
or $\Delta f=f_{m+1, n}-f_{m, n}=v_{A 0} / q_{0} R=150 k H z$.. Finding the eigenmode structure requires numerical solution and was studied in Ref. [13]. Main damping of GAE's is due to the interaction with the continuum on radial tail of the eigenmode structure which propagates to the $m+1$ branch of the Alfvén continuum [16].

\section{CAE/GAE Cyclotron excitation by beam ions}

The perturbation theory expression for the growth rate of the cyclotron instability driven by fast beam ions was derived in Ref. [17]. One can show that the strongest instability has $l \neq 0$ in the resonance condition $\mathcal{R} \equiv \omega-l \omega_{c b}-k_{\|} v_{\|}=0$. Introduce a new function $g$ for CAE $g \equiv$ $\left(J_{0}-J_{2}\right)^{2}$ and for GAE $g \equiv\left(J_{0}+J_{2}\right)^{2}$. In the case of co-injection we have to choose $l=-1$ and $\omega<0$ to match the frequency of the measured and predicted GAE spectra. Typically the distribution function can be expressed as a sum of two parts: (1) almost tangentially confined passing with narrow width in pitch angle $\lambda, f_{b p}=3 B^{2} \beta_{b}(1-\eta) e^{-\lambda / \delta \lambda_{p}} / v^{3} \delta \lambda_{p}^{2} 2^{3} \pi E_{b 0}$ and (2) trapped bump-on-tail in $\lambda$ direction, which we assume as Gaussian with narrow width $\delta \lambda_{t} \ll 1, f_{b t}=3 B^{2} \beta_{b} \eta \sqrt{1-\lambda_{0}^{2}} e^{-\left(\lambda-\lambda_{0}\right)^{2} / \delta \lambda_{t}^{2}} / v^{3} \delta \lambda_{t} \lambda_{0} 2^{3} \pi^{3 / 2} E_{b 0}$, where $\eta$ gives a fraction of trapped ions. Due to injection geometry for dense plasma $\eta$ can be close to one, while for low density plasma it is much smaller than 1 . In the limit $\omega^{2} / \omega_{c b}^{2} \ll 1$ the expression for GAE growth rate [4]:

$$
\frac{\gamma_{b G A E}}{\omega}=\frac{-T_{i} \beta_{b}}{E_{b 0} \beta_{i}} \frac{3 \pi \omega_{c b}\left(l \omega_{c i}+\omega\right)}{2 \omega^{2}}\left\{(1-\eta)\left[2-6 \delta \lambda_{p}^{2} \frac{\omega}{l \omega_{c b}}\right]+\eta\left[\frac{\partial\left(v_{\perp}^{2} g_{t}\right)}{v_{\perp} \partial v_{\perp}}-\frac{\omega \lambda_{0}^{2} g_{t}}{l \omega_{c b}}\right]\right\},
$$

where $g_{t}=g\left(\lambda=\lambda_{0}\right)$ is relevant for trapped particles. For CAEs the growth rate expression is similar $\gamma_{b C A E} \simeq \gamma_{b G A E}$, whereas $g_{t}$ function is to be taken according to CAE resonance condition. The integrand determines conditions for the instability. The trapped ion driving term $\left(\sim g^{\prime}\right)$ is dominant in Eq. (2). Analysis shows [4] that for the instability one should have $1<\left(-\omega / \omega_{c b}\right)\left(v_{\perp b 0} / v_{A}\right)<2$ for CAEs and $2<\left(-\omega / \omega_{c b}\right)\left(v_{\perp b 0} / v_{A}\right)\left(k_{\perp} / k_{\|}\right)<4$ for GAE's. Note that the finite width of the distribution function in $v_{\perp}$ (i.e. $\delta \lambda_{t}$ ) is stabilizing. Approximate requirements for such stabilization can be obtained from function $g_{t}$, so that for CAEs one needs $\delta \lambda_{t} v>-v_{A} \omega_{c b} / \omega$, and for GAE's $\delta \lambda_{t}>-2 v_{A} \omega_{c b} / \omega$. These expressions give growth rates on the order of $\gamma_{b} /|\omega| \simeq n_{b t} / n_{i} \leq 1 \%$, where $n_{b t}$ is the characteristic density of trapped beam ions. The term due to passing particles in the expression for the growth rate can be driving if the width of the passing particle distribution function is $\delta \lambda_{p}^{2}>-\omega_{c b} / 3 \omega$. Typically the distribution function of beam ions becomes isotropic below the critical energy so that the drive is possible within the energy range $E_{b *}<E<E_{b 0}$ or in NSTX $20 \mathrm{keV}<E<80 \mathrm{keV}$ , i.e. $v_{b 0} / 2<v<v_{b 0}$. Trapped particles can be in the resonance if their parallel velocity satisfies $v_{\|} / v_{A}>\left(1+\omega_{c b} / \omega\right)$ for GAE's and $v_{\|} / v_{A}>k_{\perp}\left(1+\omega_{c b} / \omega\right) / k_{\|}$for CAEs.

\section{Damping rates}

The damping rate for CAE modes in the frequency range of interest comes from electron Landau damping. It can be avoided if the phase velocity of CAE is smaller or bigger than the electron thermal velocity. For example (see Ref. [3]) with the damping rate bounded by $\gamma_{d} / \omega<1 \%$ one finds two domains for $k_{\|}: \zeta_{e} \equiv\left(k_{\perp} / k_{\|}\right) \sqrt{m_{e} / m_{i} \beta_{e}}>1.5$ or $\zeta_{e}<0.45$. The first condition is typically satisfied in tokamaks and in STs, whereas the second one is true for STs due to large electron beta.

GAE main damping mechanism is so-called continuum damping, which is due to mode coupling to the kinetic shear Alfvén wave at the edge of the plasma. It was studied numerically in Ref. [16]. Perturbative theory was applied to evaluate continuum damping in Ref. [4] and 
the following expression was obtained

$$
\frac{\gamma_{d}}{\left|\omega_{0}\right|}=-\frac{\pi}{4} O(1) \frac{x_{2}^{2 m+\delta}}{x_{s}^{2 m+2 \delta}} \frac{1.95 m^{2 / 3}}{\left(m^{2 / 3}+5 / 3\right)},
$$

where $x_{2}$ is the right boundary of the GAE domain, $x_{s} \geq x_{2}$ is the minor radius at which GAE interacts with the continuum, and $\delta \geq 2$ is the parameter, which controls the continuum profile. It is clear that this contribution is always stabilizing. One can notice that as long as $x_{2}<x_{s}$, i.e. the singularity is outside the mode localization, high- $m$ modes are weakly damped, $\gamma_{d} /\left|\omega_{0}\right| \sim\left(x_{2} / x_{s}\right)^{2 m+\delta}$. The maximum continuum damping $\left(\gamma_{d} /\left|\omega_{0}\right|\right)_{\max } \sim 1$ is achieved when the singular surface is within the mode localization, that is $x_{2}=x_{s}$. In this case our perturbative technique is not valid. Low poloidal mode number GAEs $m \leq 2$ should suffer stronger damping from the continuum due to their global structure extended enough to stabilize the beam ion drive.

\section{Conclusions}

High frequency modes with frequencies below the fundamental cyclotron frequency of thermal ions were observed in NSTX. Based on the measured spectrum of high frequency modes we identify them as CAEs and GAE's. CAEs have similar time evolution as plasma parameters change, while GAE's may intersect due to $q$-profile relaxation. A theory has been developed to study the properties of these mode. Both types of instabilities are driven by the tangential NBI in NSTX. Beam ions excite CAE/GAE's through the Doppler shifted cyclotron resonance. The main source for the drive is the velocity space anisotropy of the beam ion distribution function. Simulations of the effect CAE/GAE's may have on plasma ions indicate that these modes may provide a channel for efficient energy transfer from fast ions directly to thermal ions [18].

\section{References}

[1] FREDRICKSON, E., et. al., Phys. Rev. Lett. 87 (2001) 145001.

[2] FREDRICKSON, E., et. al., Phys. Plasmas 9 (2002) 2069.

[3] GORELENKOV, N. N.,et. al., Nucl.Fusion 42 (2002) 977.

[4] GORELENKOV, N. N., et. al., Nucl.Fusion 43 (2003).

[5] GORELENKOV, N. N., et. al., Phys. Plasmas 9 (2002) 3483.

[6] GREENE, G. J., and the TFTR Group, in Proceedings of 17th European Conference on Controlled Fusion and Plasma Heating, Amsterdam, 1990, Part IV, Vol. 14B, p.1540.

[7] COTTRELL, G. A., DENDY, R. O. , Phys. Rev. Lett. 6033 (1988).

[8] MAHAJAN, S. M., and Ross, D. W., Phys. Fluids 262561 (1983).

[9] COPPI, B., et.al., Phys. Fluids 294060 (1986).

[10] GORELENKOV, N. N., and CHENG, C. Z., Nucl. Fusion 35 (1995) 1743.

[11] FÜLŐP, T., et.al., Nucl. Fusion 371281 (1997).

[12] KOLESNICHENKO, Ya., et.al., Nucl. Fusion 381871 (1998).

[13] APPERT, K., et. al., Plasma Physics 24 (1982) 1147.

[14] BELOVA E. V. et al., Phys. Plasmas 7 (2000) 4996. BELOVA E. V., et.al., "Numerical simulations of CAE modes in NSTX", Bull. Am. Phys. Soc. v. 46, 334 (2001)

[15] CHENG, C. Z. , Phys. Reports 211 (1992) 1.

[16] VAN DAM, J.W., et. al., Fusion Technology 18 (1990) 461.

[17] GORELENKOV, N. N., and CHENG, C. Z., Phys. Plasmas 2 (1995) 1961.

[18] GATES, D. A., et. al., Phys. Rev. Lett. 87 (2001) 205003. 


\section{External Distribution}

Plasma Research Laboratory, Australian National University, Australia

Professor I.R. Jones, Flinders University, Australia

Professor João Canalle, Instituto de Fisica DEQ/IF - UERJ, Brazil

Mr. Gerson O. Ludwig, Instituto Nacional de Pesquisas, Brazil

Dr. P.H. Sakanaka, Instituto Fisica, Brazil

The Librarian, Culham Laboratory, England

Mrs. S.A. Hutchinson, JET Library, England

Professor M.N. Bussac, Ecole Polytechnique, France

Librarian, Max-Planck-Institut für Plasmaphysik, Germany

Jolan Moldvai, Reports Library, Hungarian Academy of Sciences, Central Research Institute for Physics, Hungary

Dr. P. Kaw, Institute for Plasma Research, India

Ms. P.J. Pathak, Librarian, Institute for Plasma Research, India

Ms. Clelia De Palo, Associazione EURATOM-ENEA, Italy

Dr. G. Grosso, Instituto di Fisica del Plasma, Italy

Librarian, Naka Fusion Research Establishment, JAERI, Japan

Library, Laboratory for Complex Energy Processes, Institute for Advanced Study, Kyoto University, Japan

Research Information Center, National Institute for Fusion Science, Japan

Dr. O. Mitarai, Kyushu Tokai University, Japan

Dr. Jiangang Li, Institute of Plasma Physics, Chinese Academy of Sciences, People's Republic of China

Professor Yuping Huo, School of Physical Science and Technology, People's Republic of China

Library, Academia Sinica, Institute of Plasma Physics, People's Republic of China

Librarian, Institute of Physics, Chinese Academy of Sciences, People's Republic of China

Dr. S. Mirnov, TRINITI, Troitsk, Russian Federation, Russia

Dr. V.S. Strelkov, Kurchatov Institute, Russian Federation, Russia

Professor Peter Lukac, Katedra Fyziky Plazmy MFF UK, Mlynska dolina F-2, Komenskeho Univerzita, SK-842 15 Bratislava, Slovakia

Dr. G.S. Lee, Korea Basic Science Institute, South Korea

Institute for Plasma Research, University of Maryland, USA

Librarian, Fusion Energy Division, Oak Ridge National Laboratory, USA

Librarian, Institute of Fusion Studies, University of Texas, USA

Librarian, Magnetic Fusion Program, Lawrence Livermore National Laboratory, USA

Library, General Atomics, USA

Plasma Physics Group, Fusion Energy Research Program, University of California at San Diego, USA

Plasma Physics Library, Columbia University, USA

Alkesh Punjabi, Center for Fusion Research and Training, Hampton University, USA

Dr. W.M. Stacey, Fusion Research Center, Georgia Institute of Technology, USA

Dr. John Willis, U.S. Department of Energy, Office of Fusion Energy Sciences, USA

Mr. Paul H. Wright, Indianapolis, Indiana, USA 
The Princeton Plasma Physics Laboratory is operated by Princeton University under contract with the U.S. Department of Energy.

\author{
Information Services \\ Princeton Plasma Physics Laboratory \\ P.O. Box 451 \\ Princeton, NJ 08543
}

Phone: 609-243-2750

Fax: 609-243-2751

e-mail: pppl_info@pppl.gov

Internet Address: http://www.pppl.gov 